\title{
GLOBAL EXTRAPOLATION OF A FIRST ORDER SPLITTING METHOD*
}

\author{
J. G. VERWER † AND H. B. DE VRIES $\ddagger$
}

\begin{abstract}
This note deals with the numerical solution of multi-space dimensional parabolic partial differential equations and advocates classic global Richardson extrapolation for splitting methods. The paper concentrates on the first order locally one-dimensional splitting method. This robust, low order splitting method is known to possess two favourable properties. It rapidly damps high frequency components, which is of importance for problems having nonsmooth initial data, and it possesses excellent stability properties for nonlinear problems. Global extrapolation to higher order leaves these properties invariant. In addition, global extrapolation is easy to implement. A comparison is made with a local extrapolation procedure which has been proposed by Lawson and Morris (SIAM J. Numer. Anal., 15 (1978), pp. 1212-1224). A few numerical results are reported.
\end{abstract}

AMS(MOS) 1980 subject classifications. 65L05, 65M05, 65M20

1982 CR categories. 5.17

Key words. numerical analysis, parabolic partial differential equations, splitting methods, method of lines, global extrapolation

1. Introduction. In the numerical solution of parabolic partial differential equations with nonsmooth initial data, it is desirable to employ a time discretization which, in a sufficient manner, simulates the rapid exponential decay of high frequency components. For example, when a discontinuity exists between the initial function and the boundary conditions. Within the class of splitting methods for multi-space dimensional problems, the first order locally one-dimensional method (LOD method, cf. [17]) possesses this damping property. A disadvantage of this method is its low accuracy in time. To increase the accuracy, Lawson and Morris [9] have proposed a local extrapolation of the LOD method to order two which maintains the rapid damping of high frequency components. ${ }^{1}$ This locally extrapolated LOD scheme requires in general twice as many operations per step as the basic LOD scheme. However, a numerical experiment [9] on a model heat equation in two space dimensions with a discontinuity between the initial and boundary conditions, has shown that local extrapolation may pay off. In particular, for such problems the Lawson-Morris scheme will perform better than the second order Peaceman-Rachford scheme due to a lack of damping of high frequency components in the latter one.

We advocate an alternative extrapolation of the LOD method, viz. global Richardson extrapolation. This type of Richardson extrapolation, which is classic in the numerical solution of ordinary differential equations [5, p. 81], involves parallel integration with the same basic scheme on different time grids, but completely separated. Consequently, all stability and damping properties of the basic scheme are left invariant by global extrapolation, contrary to local extrapolation. Global extrapolation is easy to implement. For the LOD method it is also less expensive than the Lawson-Morris extrapolation. Global extrapolation to order two requires one and a half-times as many operations per step as the basic scheme. For global extrapolation to order three this factor is equal to $7 / 4$ or 2 , depending on the implementation.

*Received by the editors March 29, 1983, and in revised form May 17, 1984.

†Centre for Mathematics and Computer Science, Foundation Mathematical Centre, Kruislaan 413, 1098 SJ Amsterdam, the Netherlands.

$\ddagger$ Fokker, B.V., Dept. RAMS, S018-29, 1117 ZJ Schiphol Oost, the Netherlands.

'As a sequel to [9] Gourlay and Morris have published [3] and [4] where they develop new schemes of orders 2,3 and 4 which also rapidly damp high frequency components. However, in contrast to those of [9], no splitting versions of their new schemes were given and, as far as we know, the question of applying them in a splitting context has not been discussed in the literature. 
Recent alternative approaches to increasing the accuracy of splitting methods include the application of defect correction techniques [7], [8], [14]. A common property of these techniques with the local extrapolation technique of Lawson and Morris [9], is that the accuracy is increased by some local procedure. For splitting methods such local procedures always seem to interfere with the requirement of unconditional stability and, particularly, with rapid damping of high frequency components. In this respect, the present approach differs in principle. By global extrapolation the accuracy is increased in a global way and by no means influences the stepwise stability of the solution process. This latter point is our main motive to advocate global extrapolation. Furthermore, the technique is simple and can be applied to any one-step splitting method for time-dependent, multi-space dimensional problems.

In this note we concentrate on the LOD method. Apart from its well-known damping properties, which is an essential requirement in [9], this method also possesses excellent stability properties for nonlinear problems [15]. Global extrapolation does not interfere with these nonlinear stability properties either. Furthermore, the LOD method is not restricted to two space dimensions; it is equally applicable to two- and three-space dimensional problems.

2. The LOD method. In this section we briefly recall the LOD method [17]. By following the method of lines approach, the LOD method can be formulated in a very compact way (cf. [6]). Let the initial value problem for the ordinary differential system

$$
\dot{y}(t)=f(t, y(t)), \quad t>0, \quad y(0)=y_{0}
$$

represent a semi-discrete version of a given initial-boundary value problem for a partial differential equation. For the moment it will not be necessary to be specific about the partial differential equation and the space discretization. We only assume that the vector function $f(t, y)$ can be written as

$$
f(t, y)=\sum_{i=1}^{k} f_{i}(t, y),
$$

where $f_{i}$ corresponds to a one-space dimensional partial differential operator. The following time integration formula:

$$
\begin{aligned}
& y_{n+1}^{(0)}=y_{n}, \\
& y_{n+1}^{(i)}=y_{n+1}^{(i-1)}+\tau f_{i}\left(t_{n+1}^{(i)}, y_{n+1}^{(i)}\right), \quad i=1, \cdots, k, \\
& y_{n+1}=y_{n+1}^{(k)},
\end{aligned}
$$

then defines the LOD step $y_{n} \rightarrow y_{n+1}$. Here, $y_{n}$ is the approximation to $y(t)$, the exact solution of (2.1), at time $t=t_{n}$, and $\tau=t_{n+1}-t_{n}$ is the timestep. Further, we suppose $t_{n} \leqq t_{n+1}^{(i)} \leqq t_{n+1}$, $i=1, \cdots, k$.

In applications $k$ is normally equal to 2 or 3 , being the space dimension of the partial differential equation. In $\S 4$ we shall give a numerical example of a two- and three-space dimensional problem. Note that we formulate the splitting method directly for time-dependent and, possibly, nonlinear problems.

The order of consistency of (2.3) is equal to one for all splitting functions $f_{i}, i=1, \cdots, k$, satisfying (2.2). Observe that (2.3) consists of $k$ consecutive backward Euler steps, each of which applied with a different function $f_{i}$. The computation of the vectors $y_{n+1}^{(i)}$ from the implicit backward Euler relations can be performed cheaply using a Newton type iteration, because we assumed that $f_{i}$ stands for a semi-discrete, one-space dimensional partial differential operator. 
The LOD method is known to be unconditionally stable for the linear model problem

$$
U_{t}=\sum_{i=1}^{k} U_{x_{i} x_{i}}
$$

where the second order derivative has been replaced by the central difference operator.

Furthermore, the method possesses excellent damping properties for high frequency solution components (see e.g. [9]). In addition to these linear stability properties, the LOD method can also be shown to be unconditionally stable for nonlinear parabolic problems of the form

$$
U_{t}=\sum_{i=1}^{k} F_{i}\left(t, x, U, \frac{\partial}{\partial x_{i}}\left(p_{i}(t, x) \frac{\partial U}{\partial x_{i}}\right)\right), \quad x \in \Omega \subset \mathbb{R}^{k} .
$$

This nonlinear stability result can be shown by exploiting the intimate relation with the backward Euler scheme. For details we refer to [15].

To conclude our description of the LOD method we have to recall two possible sources of inaccuracies, viz. nonconstant boundary values and nonconstant inhomogeneous terms [2], [12], [16]. These inaccuracies will also be noticeable for locally and globally extrapolated results (see Experiment 2 of $\$ 5$ ). Other splitting methods, such as ADI, also suffer from these phenomena, although to a somewhat lesser extent.

3. Global Richardson extrapolation. The LOD method (2.3) may be considered as a particular one-step integration method of order of consistency $p=1$ for the ordinary differential system (2.1). Suppose that a $p$ th order one-step method is applied from $t_{0}=0$ up to $t_{N}=T$, using a time grid $G_{N}$. This grid does not need to be uniform. It is only assumed that for $N$ sufficiently large, the minimal and maximal stepsizes $\tau$ behave like $O\left(N^{-1}\right)$. If this natural assumption is satisfied, we are assured of the existence of an asymptotic expansion in the maximal stepsize, $\tau_{N}$ say, for the global error [13]

$$
\varepsilon_{N}=y_{N}-y\left(t_{N}\right) .
$$

If we let $f$ be $M$ times differentiable, in some neighbourhood of the exact solution (2.1), then functions $e_{j}, j=p, \cdots, M$, exist, independent of $\tau_{N}$, such that

$$
\varepsilon_{N}=\sum_{j=p}^{M} \tau_{N}^{j} e_{j}\left(t_{N}\right)+O\left(\tau_{N}^{M+1}\right), \quad \tau_{N} \rightarrow 0
$$

The existence of this asymptotic expansion for $\varepsilon_{N}$ forms the basis for global Richardson extrapolation.

The use of this technique for estimating the global error of one-step integration methods for ordinary differential equations is classic (see [5, p. 81] and [13, p. 157]), although it is not very often applied [1], [10], [11]. As far as we know, the possibility of using this technique for increasing the accuracy of low order splitting methods has not yet been discussed in the literature.

Global extrapolation is easy to implement. It involves parallel integration with the same method on different grids $G_{N}$. Let us consider the coherent grids $G_{N}, G_{2 N}$ and $G_{3 N}$ depicted in Fig. 1. $G_{2 N}$ is obtained from $G_{N}$ by halving all stepsizes, etc. Because of this coherence between the grids, expansion (3.2) holds for $\tau_{N}, \tau_{2 N}=\tau_{N} / 2$ and $\tau_{3 N}=\tau_{N} / 3$, at all common gridpoints, i.e., on the whole of $G_{N}$. Let $y_{n, i}$ denote the approximation to $y\left(t_{n}\right)$ at the grid $\boldsymbol{G}_{i N}$. Then, at all common points,

$$
\varepsilon_{n, i} \equiv y_{n, i}-y\left(t_{n}\right)=\sum_{j=1}^{M}\left(\tau_{N} / i\right)^{j} e_{j}\left(t_{n}\right)+O\left(\tau_{N}^{M+1}\right), \quad \tau_{N} \rightarrow 0,
$$


if $p=1$. Next suppose $M$ sufficiently large and compute

$$
\begin{aligned}
y_{n}^{|2|} & \equiv 2 y_{n, 2}-y_{n, 1}, \\
y_{n}^{|3|} & \equiv \frac{9}{2} y_{n, 3}-4 y_{n, 2}+\frac{1}{2} y_{n, 1} .
\end{aligned}
$$

Then

$$
\begin{aligned}
& y_{n}^{|2|}=y\left(t_{n}\right)-\frac{1}{2} \tau_{N}^{2} e_{2}\left(t_{n}\right)+O\left(\tau_{N}^{3}\right), \\
& y_{n}^{|3|}=y\left(t_{n}\right)+\frac{1}{6} \tau_{N}^{3} e_{3}\left(t_{n}\right)+O\left(\tau_{N}^{4}\right),
\end{aligned}
$$

showing that $y_{n}^{[2]}$ and $y_{n}^{[3]}$ are of order of convergence two and three, respectively.

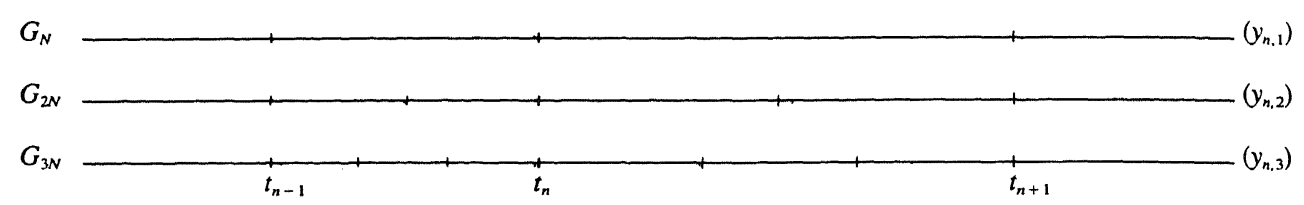

FIG. 1. Three coherent grids.

It is emphasized that the extrapolation is passive, i.e, the integrations are performed independently of each other. This trivially implies that global extrapolation cannot interfere with the stability of the basic scheme.

Observe that it is theoretically possible to extrapolate to arbitrarily high order of convergence. We restrict ourselves to orders two and three, assuming that this is sufficiently high for partial differential equations. When compared with the result $y_{n, 2}$ computed at the grid $G_{2 N}$, the computation of $y_{n}^{[2]}$ requires an additional computational effort of $50 \%$. Global extrapolation to order three requires twice as many operations per step as the basic scheme on $G_{3 N}$. When using the grids $G_{N}, G_{2 N}$ and $G_{4 N}$, the corresponding factor is equal to $7 / 4$. We prefer to use $G_{N}, G_{2 N}$ and $G_{3 N}$ in order to avoid a too large difference between the stepsizes.

4. Comparison with the Lawson-Morris extrapolation scheme. Lawson and Morris [9] restrict their investigations to constant coefficient, homogeneous linear semi-discrete systems

$$
\dot{y}=A y=\sum_{i=1}^{k} A_{i} y,
$$

where $1 \leqq k \leqq 3$. For this linear problem, the LOD scheme (2.3) reduces to

$$
y_{n+1}=\prod_{i=k}^{1}\left(I-\tau A_{i}\right)^{-1} y_{n} .
$$

The second order, locally extrapolated LOD scheme of [9] is given by

$$
\begin{gathered}
z_{0}=\prod_{i=k}^{1}\left(I-\tau A_{i}\right)^{-1} y_{n}, \quad z_{1}=\prod_{i=1}^{k}\left(I-\tau A_{i}\right)^{-1} z_{0}, \\
z_{2}=\prod_{i=k}^{1}\left(I-2 \tau A_{i}\right)^{-1} y_{n}, \quad z_{3}=\prod_{i=1}^{k}\left(I-2 \tau A_{i}\right)^{-1} y_{n}, \\
y_{n+2}=2 z_{1}-\frac{1}{2}\left(z_{2}+z_{3}\right) .
\end{gathered}
$$


This scheme performs the step $y_{n} \rightarrow y_{n+2}$ over an interval of length $2 \tau$ by performing four basic LOD steps. Hence, per interval of length $\tau$, it requires twice as many operations as the basic scheme. Lawson and Morris have defined their extrapolation in such a way that for the linear model (4.1) associated to (2.4) (i) the scheme is unconditionally stable, and (ii) the damping of the basic scheme is maintained. In fact, the preservation of damping has been their main concern.

Remark. The reader should observe the symmetrization in method (4.3), i.e., the order in which the matrices $A_{i}$ appear alternates. Lawson and Morris do not motivate their use of symmetrization. However, it benefits the accuracy and stability. We also remark that symmetrization is not necessary for obtaining order two. Further, if the matrices commute, which they do in the stability analysis, we get $y_{n+2}=2 z_{1}-z_{2}$, i.e., straightforward local Richardson extrapolation to order two. Hence in this case the extrapolation requires $3 / 2$ times as many operations. If the matrices do commute we use this work estimate for scheme (4.3) rather than the factor 2 mentioned above.

The second order, local extrapolation scheme (4.3) can be extended to the general problem cases (2.1)-(2.2). Introduce the formal notation $y_{n+1}=E\left[\tau, t_{n}, y_{n}, f_{1}, \cdots, f_{k}\right]$ for the LOD formula (2.3). The extention then reads

$$
\begin{gathered}
z_{0}=E\left[\tau, t_{n}, y_{n}, f_{1}, \cdots, f_{k}\right], \quad z_{1}=E\left[\tau, t_{n+1}, z_{0}, f_{k}, \cdots, f_{1}\right], \\
z_{2}=E\left[2 \tau, t_{n}, y_{n}, f_{1}, \cdots, f_{k}\right], \quad z_{3}=E\left[2 \tau, t_{n}, y_{n}, f_{k}, \cdots, f_{1}\right], \\
y_{n+2}=2 z_{1}-\frac{1}{2}\left(z_{2}+z_{3}\right) .
\end{gathered}
$$

A straightforward Taylor expansion shows that this algorithm is of second order for any choice of intermediate time points $t_{n+1}^{(i)}$ in (2.3).

To demonstrate their scheme, Lawson and Morris [9] computed the solution to the problem

$$
\begin{aligned}
& U_{t}=U_{x_{1} x_{1}}+U_{x_{2} x_{2}}, \quad t>0, \quad 0<x_{1}, x_{2}<2, \\
& U\left(0, x_{1}, x_{2}\right)=\sin \left(\frac{\pi x_{2}}{2}\right), \quad 0 \leqq x_{1}, x_{2} \leqq 2, \\
& U\left(t, x_{1}, x_{2}\right)=0, \quad t>0, \quad x_{1}, x_{2} \text { on the boundary. }
\end{aligned}
$$

The Fourier solution of this problem is given by

$$
U\left(t, x_{1}, x_{2}\right)=\sin \left(\frac{\pi x_{2}}{2}\right) \sum_{n=1}^{\infty}\left[1-(-1)^{n}\right] \frac{2}{n \pi} \sin \left(\frac{n \pi x_{1}}{2}\right) \exp \left(-\frac{\pi^{2}}{4}\left(n^{2}+1\right) t\right)
$$

Because of the discontinuity between the initial function and the boundary function, this problem should be integrated with a method which rapidly damps high frequency components.

Following Lawson and Morris [9], we also compute the solution to this problem, at $t=1$, using the first order LOD scheme, the second order Lawson-Morris scheme, the second order ADI scheme of Peaceman-Rachford, and global extrapolation to order two and three applied to the LOD scheme. The spatial discretization is based on the standard 5point finite difference operator on a uniform grid of stepsize $h$. In Table 2 we show the maximum of the absolute errors for some values of $\tau$ and $h$. It should be noted that at $t=1$ the theoretical solution has a maximum value of approximately 0.01 . We refer to [9] for a plot of this two-dimensional function. The values of $\tau$ given in the table belong to the finest time grids. In the column "Work" we have expressed the total computational effort per $\tau$ interval into the computational effort of the LOD scheme. 
TABLE 2

Maximum absolute errors in solving problem (4.5) at $t=1$.

\begin{tabular}{l|c|ccc|ccc}
\hline \multirow{2}{*}{ Method } & & \multicolumn{3}{c|}{$\tau=1 / 12$} & \multicolumn{3}{c}{$\tau=1 / 24$} \\
& Work & $h=0.1$ & $h=0.05$ & $h=0.025$ & $h=0.1$ & $h=0.05$ & $h=0.025$ \\
\hline LOD & 1 & $5.3_{10^{-3}}$ & $5.2_{10^{-3}}$ & $5.2_{10^{-3}}$ & $2.5_{10^{-3}}$ & $2.5_{10^{-3}}$ & $2.5_{10^{-3}}$ \\
Peaceman-Rachford & $3 / 2$ & $2.3_{10^{-3}}$ & $1.8_{10^{-2}}$ & $4.1_{10^{-2}}$ & $3.4_{10^{-5}}$ & $2.3_{10^{-3}}$ & $1.8_{10^{-2}}$ \\
Lawson-Morris & $3 / 2$ & $7.5_{10^{-4}}$ & $6.9_{10^{-4}}$ & $6.7_{10^{-4}}$ & $3.0_{10^{-4}}$ & $2.4_{10^{-4}}$ & $2.3_{10^{-4}}$ \\
Global extrap. (2) & $3 / 2$ & $8.4_{10^{-4}}$ & $9.0_{10^{-4}}$ & $9.2_{10^{-4}}$ & $1.8_{10^{-4}}$ & $2.3_{10^{-4}}$ & $2.5_{10^{-4}}$ \\
Global extrap. (3) & 2 & $5.5_{10^{-5}}$ & $1.1_{10^{-4}}$ & $1.2_{10^{-4}}$ & $5.9_{10^{-5}}$ & $3.1_{10^{-6}}$ & $1.1_{10^{-5}}$ \\
\hline
\end{tabular}

It can be concluded that for problem (4.5), which serves as a test example for problems with nonsmooth initial data, the third order global extrapolation scheme is to be preferred to the other schemes. For example, the globally extrapolated third order results for $\tau=1 / 12$ are more accurate than the second order extrapolated results for $\tau=1 / 24$. Note the somewhat awkward error behaviour of the third order results for the three different values of $h$. It seems plausible to owe this to interference of space and time errors of nearly equal magnitude. We recall that Table 2 shows full discretization errors, whereas the implemented extrapolations only deal with the time integration. We also emphasize that the very minor increasing error behaviour of the global extrapolation methods for decreasing $h$ does not occur systematically. See Tables $3 a, b$ for comparison. Finally it should be observed that the ADI scheme yields relatively large errors when $h$ decreases. This is caused by a lack of damping of high frequency components. The LOD type schemes do not suffer from this phenomenon.

TABLE 3a

Maximum absolute errors in solving problem (4.7) at $t=1$ for $\gamma=1 / 6$.

\begin{tabular}{l|c|ccc|ccc}
\hline \multirow{2}{*}{ Method } & \multirow{3}{*}{$\begin{array}{c}\tau=1 / 12 \\
h=0.05\end{array}$} & $h=0.025$ & $h=0.1$ & $h=0.05$ & $h=0.025$ \\
\hline LOD & 1 & $3.7_{10^{-3}}$ & $3.4_{10^{-3}}$ & $3.4_{10^{-3}}$ & $1.9_{10^{-3}}$ & $1.7_{10^{-3}}$ & $1.7_{10^{-3}}$ \\
Lawson-Morris & $3 / 2$ & $6.3_{10^{-4}}$ & $4.0_{10^{-4}}$ & $3.4_{10^{-4}}$ & $4.1_{10^{-4}}$ & $1.8_{10^{-4}}$ & $1.2_{10^{-4}}$ \\
Global extrap. (3) & 2 & $2.5_{10^{-4}}$ & $3.7_{10^{-5}}$ & $3.0_{10^{-5}}$ & $2.9_{10^{-4}}$ & $6.9_{10^{-5}}$ & $1.3_{10^{-5}}$ \\
\hline
\end{tabular}

TABLE $3 b$

Time integration errors at the gridpoint $(1 / 2,1 / 2,1 / 2)$.

\begin{tabular}{l|rr}
\hline & $\tau=1 / 12$ & $\tau=1 / 24$ \\
\hline LOD & $-3.4_{10^{-3}}$ & $-1.6_{10^{-3}}$ \\
Lawson-Morris & $-3.3_{10^{-4}}$ & $-1.0_{10^{-4}}$ \\
Global extrap. (3) & $5.0_{10^{-5}}$ & $6.0_{10^{-6}}$ \\
\hline
\end{tabular}

As a further illustration we also compute the solution to the following three-dimensional version of problem (4.5):

$$
\begin{aligned}
& U_{t}=\gamma\left(U_{x_{1} x_{1}}+U_{x_{2} x_{2}}+U_{x_{3} x_{3}}\right), \quad t>0, \quad 0<x_{1}, x_{2}, x_{3}<1, \\
& U\left(0, x_{1}, x_{2}, x_{3}\right)=\sin \left(\pi x_{2}\right) \sin \left(\pi x_{3}\right), \quad 0 \leqq x_{1}, x_{2}, x_{3} \leqq 1, \\
& U\left(t, x_{1}, x_{2}, x_{3}\right)=0, \quad t>0, \quad x_{1}, x_{2}, x_{3} \text { on the boundary. }
\end{aligned}
$$


The Fourier solution is given by

$$
U\left(t, x_{1}, x_{2}, x_{3}\right)=\sin \left(\pi x_{2}\right) \sin \left(\pi x_{3}\right) \sum_{n=1}^{\infty}\left[1-(-1)^{n}\right] \frac{2}{n \pi} \sin \left(n \pi x_{1}\right) \exp \left(-\gamma \pi^{2}\left(n^{2}+2\right) t\right)
$$

Again we have a discontinuity between the initial and boundary function. The spatial discretization is based on the standard 7-point finite difference operator on a uniform grid of stepsize $h$. Table 3 a shows the maximum of the absolute errors for some values of $\tau$ and $h$ at $t=1$ for three LOD schemes using $\varphi=1 / 6$. For this value of $\gamma$ the solution (4.8) has a maximum value of approximately 0.01 at $t=1$. The column "Work" has the same meaning as in Table 2.

Again we may conclude that it pays to apply extrapolation. It was found that in all cases the maximum absolute error appeared near the point $(1 / 2,1 / 2,1 / 2)$. For this grid point the space errors (fully continuous solution - semi-discrete solution) are $-3.0_{10^{-4}}$, $-7.4_{10^{-5}}$ and $-1.9_{10^{-5}}$. For all three methods the corresponding time errors (semi-discrete solution - fully discrete solution) were found to be independent of $h$. They are listed in Table $3 \mathrm{~b}$. This table shows the performance of the extrapolation procedures more clearly than Table $3 \mathrm{a}$.

5. Two more numerical experiments. In addition to the previous experiments, we discuss two more experiments in order to give some more insight into the use of the extrapolation procedures. For this purpose we consider the first initial-boundary value problem for the simple linear problems

$$
\begin{aligned}
& U_{t}=\frac{x_{1}-x_{1}^{2}}{4} U_{x_{1} x_{1}}+\frac{x_{2}-x_{2}^{2}}{4} U_{x_{2} x_{2}}, \quad t>0, \quad 0<x_{1}, x_{2}<1, \\
& U\left(t, x_{1}, x_{2}\right)=1-e^{-t}\left(x_{1}^{2}-x_{1}\right)\left(x_{2}^{2}-x_{2}\right), \quad \mathrm{t} \geqq 0, \quad 0 \leqq x_{1}, x_{2} \leqq 1,
\end{aligned}
$$

and

$$
\begin{aligned}
& U_{t}=U_{x_{1} x_{1}}+U_{x_{2} x_{2}}-e^{-t}\left(x_{1}^{2}+x_{2}^{2}+4\right), \quad t>0, \quad 0<x_{1}, x_{2},<1, \\
& U\left(t, x_{1}, x_{2}\right)=1+e^{-t}\left(x_{1}^{2}+x_{2}^{2}\right), \quad t \geqq 0, \quad 0 \leqq x_{1}, x_{2} \leqq 1 .
\end{aligned}
$$

For the space discretization we use again standard finite differences on a uniform grid with meshwidth $h$. Note that, due to the solutions selected, the space discretization is exact. So we can illustrate the effect of the extrapolations without interference of space discretization errors. Note also that the selected solutions are smooth, i.e., free of high frequency components. This implies that here the ADI scheme could also successfully be applied, possibly in combination with global extrapolation.

We have applied

(i) The first order LOD scheme

$$
y^{*}=y_{n}+\tau f_{1}\left(t_{n}+\tau, y^{*}\right), \quad y_{n+1}=y^{*}+\tau f_{2}\left(t_{n}+\tau, y_{n+1}\right) .
$$

(ii) Global extrapolation on this scheme to order three.

(iii) The extension (4.4) of the Lawson-Morris scheme (4.3), where $E$ now represents (5.3).

(iv) The second order Peaceman-Rachford ADI scheme

$$
\begin{aligned}
& y^{*}=y_{n}+\frac{\tau}{2} f_{1}\left(t_{n}+\frac{1}{2} \tau, y^{*}\right)+\frac{\tau}{2} f_{2}\left(t_{n}, y_{n}\right), \\
& y_{n+1}=y^{*}+\frac{\tau}{2} f_{1}\left(t_{n}+\frac{1}{2} \tau, y^{*}\right)+\frac{\tau}{2} f_{2}\left(t_{n+1}, y_{n+1}\right) .
\end{aligned}
$$


(v) Global extrapolation on this ADI scheme to order four using the grids of Fig. 1. The extrapolation formula to order four reads

$$
y_{n}^{(4)}=\frac{27}{12} y_{n, 3}-\frac{4}{3} y_{n, 2}+\frac{1}{12} y_{n, 1} .
$$

Experiment 1. This experiment deals with the homogeneous problem (5.1). We emphasize that the solution is constant at the boundary. Space discretization leads to the splitting functions

$$
f_{1}(t, y)=A_{1} y+B_{1}, \quad f_{2}(f, y)=A_{2} y+B_{2},
$$

where $B_{1}$ and $B_{2}$ are sparse vectors containing the constant boundary values. The meaning of $A_{1}$ and $A_{2}$ should be self-evident. Because of the fact that the present semidiscrete problem is of constant coefficient type we in fact really apply the original algorithm of Lawson and Morris, as the extension of their algorithm to the inhomogeneous form is not essential.

Table 4 shows maximum absolute errors at $t=1$ for three values of $\tau$ using $h=1 / 40$. The $\tau$-values correspond to the finest grid. Observe that the LOD scheme (5.3) and the ADI scheme (5.4) were applied using half the value of $\tau$ given in the table. The column "Work" has the same meaning as in Table 2.

TABLE 4

Maximum absolute errors in solving problem (5.1) at $t=1$.

\begin{tabular}{l|c|ccc}
\hline \multicolumn{1}{c|}{ Method } & Work & $\tau=1 / 6$ & $\tau=1 / 12$ & $\tau=1 / 24$ \\
\hline LOD (applied with $\tau / 2)$ & 2 & $4.7_{10^{-4}}$ & $2.4_{10^{-4}}$ & $1.2_{10^{-4}}$ \\
Lawson-Morris & 2 & $8.0_{10^{-5}}$ & $2.3_{10^{-5}}$ & $6.2_{10^{-6}}$ \\
Global extrap. LOD (3) & 2 & $4.9_{10^{-6}}$ & $6.9_{10^{-7}}$ & $9.2_{10^{-8}}$ \\
ADI (applied with $\tau / 2)$ & 3 & $3.3_{10^{-6}}$ & $8.3_{10^{-7}}$ & $2.1_{10^{-7}}$ \\
Global extrap. ADI (4) & 3 & $2.3_{10^{-8}}$ & $1.4_{10^{-9}}$ & $8.8_{10^{-11}}$ \\
\hline
\end{tabular}

For the present problem it can be concluded that, due to the smooth solution, the homogeneity, and the constant boundary values, all methods perform relatively accurately. In particular, it pays to apply extrapolation. Also observe that the order of convergence of all time integration methods shows up clearly (the space discretization is exact).

Experiment 2. The second experiment deals with the inhomogeneous problem (5.2), whose solution has time-dependent boundary values. This experiment serves to illustrate the effect of the extrapolation procedures in the presence of time-dependent boundary values and time-dependent source terms. For all splitting methods these dependencies are known to reduce the accuracy of the time integration [2], [12], [16]. Space discretization yields the splitting functions

$$
f_{1}(t, y)=A_{1} y(t)+B_{1}(t)+{ }^{1} / 2 V(t), \quad f_{2}(t, y)=A_{2} y(t)+B_{2}(t)+1 / 2 V(t),
$$

where $V(t)$ originates from the source term of (5.2) and $A_{1}, A_{2}$ and $B_{1}(t), B_{2}(t)$ have the same meaning as in Experiment 1. The results for $h=1 / 40$ are given in Table 5. Observe that we now apply an extension of the original Lawson-Morris algorithm (4.3).

It is striking that all LOD methods are significantly less accurate than in the previous experiment, although the solution of problem (5.2) has the same "smoothness properties" as the solution of problem (5.1). For the LOD methods the order of convergence shows up insufficiently for problem (5.2). Here we must conclude that the LOD extrapolation procedures are hardly more efficient than their respective basic schemes. The errors of the 
TABLE 5

Maximum absolute errors in solving problem (5.2) at $t=1$.

\begin{tabular}{l|c|ccc}
\hline \multicolumn{1}{c|}{ Method } & Work & $\tau=1 / 6$ & $\tau=1 / 12$ & $\tau=1 / 24$ \\
\hline LOD (applied with $\tau / 2)$ & 2 & $1.2_{10^{-2}}$ & $7.8_{10^{-3}}$ & $4.5_{10^{-3}}$ \\
Lawson-Morris extension & 2 & $2.3_{10^{-2}}$ & $1.5_{10^{-2}}$ & $8.7_{10^{-3}}$ \\
Global extrap. LOD (3) & 2 & $9.8_{10^{-3}}$ & $4.7_{10^{-3}}$ & $2.0_{10^{-3}}$ \\
ADI (applied with $\tau / 2)$ & 3 & $2.6_{10^{-6}}$ & $6.6_{10^{-7}}$ & $1.6_{10^{-7}}$ \\
Global extrap. ADI (4) & 3 & $3.8_{10^{-6}}$ & $2.2_{10^{-7}}$ & $1.3_{10^{-8}}$ \\
\hline
\end{tabular}

Lawson-Morris extension are even larger than the LOD $(\tau / 2)$ errors. Finally, the ADI schemes perform very satisfactorily for the present problem. Their time integration errors are rather small while their order of convergence in time is clearly visible.

As already remarked at the end of $\$ 2$ the disappointing behaviour of the LOD algorithms lies in the time dependencies of the boundary values and the source term. ADI algorithms also suffer from these dependencies, although to a lesser extent. The decrease of accuracy can be understood from a study of the error of approximation, i.e., the defect which is obtained by substituting an exact and smooth solution into the numerical scheme. When expanding this error in the time step $\tau$, any splitting gives rise to terms which cannot be combined to a higher derivative of the smooth solution, a situation which is not encountered in the application of fully implicit schemes such as backward Euler or implicit midpoint. These terms, finite difference expressions multiplied by $h^{-2}$, thus may be relatively large when compared with derivatives of an exact solution. It turns out that these terms are clearly present when the problem has time-dependent boundary values and/or source terms (for details, see [2], [12], [16]). The fact that ADI methods suffer less than LOD from this phenomenon can be explained from the consistency of the intermediate level.

It is also worthwhile to realize that the accuracy of a splitting scheme may depend significantly on the splitting of the source term. To illustrate this we have given Table 6 which shows some results of the LOD method applied to problem (5.2), when using the splitting

$$
\begin{aligned}
& f_{1}(t, y)=A_{1} y(t)+B_{1}(t)+\alpha V(t), \\
& f_{2}(t, y)=A_{2} y(t)+B_{2}(t)+(1-\alpha) V(t),
\end{aligned}
$$

for $\alpha=0,1 / 2,1$. The correct splitting of $V(t)$ appears to be the one we have used in Table 5, at least for problem (5.2). It is obvious of course that the differences shown in Table 6 penetrate into the extrapolations. For the ADI method one will find even larger differences in accuracy for $\alpha=0,1 / 2,1$.

Finally, we should like to observe that inaccuracies caused by time-dependent boundary values can be removed by applying the Fairweather-Mitchell boundary value correction [2],

TABLE 6

Maximum absolute errors in solving problem (5.2) at $t=1$ using the LOD method for three different splittings (5.7). The mesh width $h=1 / 40$.

\begin{tabular}{l|lllll}
\hline & $\tau=1 / 5$ & $\tau=1 / 10$ & $\tau=1 / 20$ & $\tau=1 / 40$ & $\tau=1 / 80$ \\
\hline$\alpha=0$ & $9.5_{10^{-2}}$ & $6.9_{10^{-2}}$ & $4.5_{10^{-2}}$ & $2.6_{10^{-2}}$ & $1.4_{10^{-2}}$ \\
$\alpha=1 / 2$ & $1.8_{10^{-2}}$ & $1.3_{10^{-2}}$ & $8.9_{10^{-3}}$ & $5.2_{10^{-3}}$ & $2.9_{10^{-3}}$ \\
$\alpha=1$ & $5.9_{10^{-2}}$ & $4.3_{10^{-2}}$ & $2.8_{10^{-2}}$ & $1.6_{10^{-2}}$ & $8.5_{10^{-3}}$ \\
\hline
\end{tabular}


TABLE 7

Maximum absolute errors in solving problem (5.2) at $t=1$ when using the boundary value correction technique and the splitting (5.6).

\begin{tabular}{l|c|ccc}
\hline \multicolumn{1}{c|}{ Method } & Work & $\tau=1 / 6$ & $\tau=1 / 12$ & $\tau=1 / 24$ \\
\hline LOD (applied with $\tau / 2)$ & 2 & $2.2_{10^{-3}}$ & $1.3_{10^{-3}}$ & $7.0_{10^{-4}}$ \\
Lawson-Morris & 2 & $2.2_{10^{-3}}$ & $1.1_{10^{-3}}$ & $5.0_{10^{-4}}$ \\
Global extrap. LOD (3) & 2 & $1.3_{10^{-3}}$ & $4.5_{10^{-4}}$ & $1.1_{10^{-4}}$ \\
ADI (applied with $\tau / 2)$ & 3 & $2.7_{10^{-6}}$ & $6.6_{10^{-7}}$ & $1.6_{10^{-7}}$ \\
Global extrap. ADI (4) & 3 & $1.3_{10^{-6}}$ & $8.3_{10^{-8}}$ & $4.7_{10^{-9}}$ \\
\hline
\end{tabular}

[12], [16]. For problem (5.2) this is illustrated by the results shown in Table 7, although the influence of the source term is still visible (this table is to be compared with Table 5). In fact, the gain in accuracy of the extrapolated LOD schemes is still not up to our expectations. The accuracy of the ADI scheme (5.4) is not improved by applying the boundary value correction. On the other hand, the global extrapolation of the ADI scheme is benefited slightly by this correction technique.

\section{REFERENCES}

[1] K. DEKKER AND J. G. Verwer, Estimating the global error of Runge-Kutta approximation, in DifferentialDifferenzengleichungen, Anwendungen und numerische Probleme, L. Collatz, G. Meinardus and W. Wetterling, eds., ISNM Series, Vol. 62, Birkhäuser, Basel-Boston-Stuttgart, 1983.

[2] G. Fairweather AND A. R. Mitchell, A new computational procedure for ADI methods, SIAM J. Numer. Anal., 4 (1967), pp. 163-170.

[3] A. R. GOURLAY AND J. LL. MORRIS, The extrapolation of first order methods for parabolic partial differential equations. II, SIAM J. Numer. Anal., 17 (1980), pp. 641-655.

[4] - Linear combinations of generalized Crank-Nicolson schemes, IMA J. Numer. Anal., 1 (1981), pp. 347-357.

[5] P. HenRICI, Discrete Variable Methods for Ordinary Differential Equations, John Wiley, New York, 1962

[6] P. J. VAN DER HOUWEN AND J. G. VERWER, One-step splitting methods for semi-discrete parabolic equations, Computing, 22 (1979), pp. 291-309.

[7] P. J. VAN DER HOUWEN, Multistep splitting methods of high order for intial value problems, SIAM J. Numer. Anal., 17 (1980), pp. 410-427.

[8] - Iterated splitting methods of high order for time dependent partial differential equations, SIAM J. Numer. Anal., 21(1984), pp. 635-656.

[9] J. D. LAWSON AND J. LL. MORRIS, The extrapolation of first order methods for parabolic partial differential equations, I, SIAM J. Numer. Anal. 15 (1978), pp. 1212-1224.

[10] F. G. LETHER, The use of Richardson extrapolation in one-step methods with variable stepsize, Math. Comp. 20, (1966), pp. 379-385.

[11] L. F. Shampine AND H. A. WATTS, Global error estimation for ordinary differential equations, ACM Trans. Math. Software, 2 (1976), pp. 172-186.

[12] B. P. SOMmeIJer, P. J. VAN DER HOUWEN AND J. G. VeRwer, On the treatment of time-dependent boundary conditions in splitting methods for parabolic differential equations, Internat. J. Numer. Meth. Engrg., 17 (1981), pp. 335-346.

[13] H. J. STETTER, Analysis of Discretization Methods for Ordinary Differential Equations, Springer-Verlag, Berlin, 1973.

[14] J. G. VERWER, The application of iterated defect correction to the LOD method for parabolic equations, BIT, 19 (1979), pp. 384-394.

[15] - Contractivity of locally one-dimensional splitting methods, Numer. Math, 44 (1984), pp. $247-259$.

[16] YE. G. D'YAKONOV, Some difference schemes for solving boundary problems, USSR Comput. Math. Math. Phys., 1 (1963), pp. 55-77.

[17] N. N. Yanenko, The Method of Fractional Steps, Springer-Verlag, Berlin, 1971. 